\title{
Testing instance models of face repetition priming
}

\author{
DENNIS C. HAY \\ Lancaster University, Lancaster, England
}

\begin{abstract}
Two experiments examining repetition priming in face recognition are reported. They employed eight rather than the more usual two presentation trials so that the prediction made by Logan's (1988) instance model of power function speedup of response time (RT) distributions could be examined. In Experiment 1, we presented the same photograph on each trial; in Experiment 2, we presented photographs of varying poses. Both experiments showed repetition priming effects for familiar and unfamiliar faces, power function speedup for both the mean and the standard deviation of RT and the power function speedup of the quantiles of the RT distributions. We argue that our findings are consistent with the predictions made by the instance model and provide an explanatory challenge for alternative theoretical approaches.
\end{abstract}

Repetition priming is the phenomenon whereby a previously processed stimulus is recognized more quickly and more accurately on a subsequent presentation. Such effects have been observed in a wide range of face recognition experiments. For example, a speedup of response latency has been observed for familiarity judgments to second and different photographs of celebrities (Bruce \& Valentine, 1985; Ellis, Young, Flude, \& Hay, 1987), occupation judgments to celebrity faces (Young, McWeeny, Hay, \& Ellis, 1986a), and the naming of briefly presented familiar-face photographs (Ellis et al., 1987). The failure to observe repetition priming for gender decisions (Ellis, Young, \& Flude, 1990) and expression judgments (Ellis et al., 1990; Young, McWeeny, Hay, \& Ellis, 1986b) led Ellis, Flude, Bruce, and Burton (1996) to draw two main conclusions about face repetition priming. First, priming effects are restricted to the parts of the faceprocessing system that respond to the identity of a face, and second, there exist two loci at which repetition priming in recognizing famous faces operates. The first locus involves the perceptual recognition of a face as familiar and is, in their view, domain specific, by which they mean that recognition is restricted to classes of stimuli for which there is a specialized recognition system (Baddeley, 1982). That is, previous exposure to a famous face will prime recognition of later presentations of the same photograph or other similar views but will fail to prime the name of that celebrity. The second locus occurs at the stage of name retrieval and is domain independent. Thus, previously reading aloud the name of a celebrity will prime the subsequent naming of a photograph of the face of that celebrity. In a series of experiments, Ellis et al. (1996)

\footnotetext{
I thank Alan Collins, Peter Morris, and Peter Walker of the Lancaster Department of Psychology for their time discussing and commenting on various drafts, and Gordon Logan and John Wixted for insightful reviews of the first submission. Correspondence concerning this article and requests for reprints should be addressed to D.H., Department of Psychology, Fylde College, Lancaster University, Bailrigg, Lancaster, LA1 4YF, England (e-mail: d.hay@lancaster.ac.uk).
}

showed that tasks that involve familiarity or occupational decisions are susceptible to the Locus 1 priming effects whereas tasks involving face naming are susceptible to Locus 2 priming.

Ellis et al. (1996) have also argued that the new data they presented were consistent with structural accounts of face repetition priming in the Burton, Bruce, and Johnston's (1990) neural network implementation based on the interactive activation and competition networks of McClelland and Rumelhart (1981). Burton et al. use the term structural to refer to models that embrace the concept of face recognition units (FRU), which are internal representations directly equivalent to the logogens proposed by Morton (1979) to explain how words are recognized. In these accounts, repetition priming occurs when the first encounter with a stimulus lowers the activation threshold of the internal representation so that less stimulus activation is required to trigger the representation on a subsequent occasion. Ellis et al. (1996) also examined an alternative theoretical account of repetition priming - namely, the episodic or instance-based account first offered by Jacoby (1983) and Jacoby and Brooks (1984). They questioned the recognition unit metaphor and demonstrated how priming effects can be explained entirely in terms of instance retrieval. In addition, they suggested that repetition priming results from a process of perceptual enhancement, in which the memory of a previous encounter with a stimulus facilitates recognition. Ellis et al. (1996) focused on one particular instance-based account, that of Logan (1990), which draws parallels between repetition priming and the development of automaticity in task performance that follows large amounts of practice. In an attempt to integrate the explanations of these two phenomena, Logan (1990) highlighted three parallels: (1) that the response time (RT) decreases that result from repetition priming and the development of automaticity are both power functions of the number of exposures; (2) that both phenomena share item specificity-that is, only prior experiences that are similar to that which is being processed are retrieved and enhance processing speed; and, (3) that 
repetition priming and automaticity both share an associative basis. Logan also proposed that repetition priming is dependent on associations between stimuli and responses, or interpretations. Ellis et al. (1996) indicated that their data and those from a number of existing studies created problems for the latter two notions. For example, the findings that the prior reading of a name primed subsequent face naming was inconsistent with Logan's (1990) definition of item specificity. If the written name and the visual appearance of a face have nothing in common, presentation of the face for naming should not activate the prior episode of reading the name. Similarly, the view of repetition priming as being dependent on the associations between stimuli and interpretations was contradicted by the Ellis et al. (1990) finding that repetition priming did not occur when subjects were asked to decide on the gender of a face or to make expression judgments. When a second judgment is made, the prior episode should be activated, which in turn should lead to perceptual enhancement.

The purpose of this article is to rigorously examine the first of Logan's (1990) parallels between automaticity and repetition priming. Perhaps the greatest strength of Logan's instance model is the set of strong predictions that concern the speedup in responses to repeated stimuli. In Logan's (1988) theory, speedup results from a processing shift. Initially, processing is based on a set of generic, nonautomatic, cognitive procedures (i.e., algorithms) that become replaced by processing that involves direct memory access of past instances. The mechanism by which this shift occurs is simply a race between the algorithmic processing and the direct memory mechanism. On any encounter, whichever finishes the race first generates the response. Initially the algorithm may be more reliable and/or faster, but as the number of instances increases, the race becomes uneven as the algorithm competes against an increasing number of instance competitors. Direct memory times speed up as the minimum retrieval time decreases as the number of instances in memory increases. This model makes a number of strong predictions that stem from mathematical simulations of the race between the algorithm and the instances. The first prediction is that performance will speed up with practice and be well fit by a power function of the form

$$
\mathrm{RT}=a+b N^{-c},
$$

where RT is the time required to complete the task; $a$ is a constant reflecting the asymptotic performance reached; $b$ is a constant reflecting the difference between the initial and asymptotic performance; $N$ is the index of practice (i.e., the number of trials); and $c$ is a constant indicating the rate of learning. This function has been shown to apply to a wide range of tasks that involve both motor and cognitive learning performance (Newell \& Rosenbloom, 1981).

The second prediction is that the variability in performance, as measured by the standard deviation of performance over trials, will also decrease with repetition and that this performance is also well fit by a power function. However, what is most surprising is that the power functions that describe mean RT performance and the variability in the RT performance as measured by the standard deviation of the RTs are predicted to have equivalent learningrate parameters. This has been proven mathematically, substantiated by simulation, and supported by empirical data (Logan, 1988). The third prediction is that the entire distribution of RTs should decrease as a power function of the number of trials. This can be examined by partitioning the distribution of each trial into quantiles. All quantiles should be well fit by power functions in which the learning-rate parameters are equivalent to one another and to those of the overall mean RT and standard deviation functions (Logan, 1992).

Because the majority of face-priming tasks have utilized a familiarity decision (i.e., asking subjects to decide whether a stimulus face is one known before the experiment), it was thought that this would be the most appropriate vehicle for investigation. Logan (1988) has shown that his model is capable of predicting the changes in RT distributions in experiments using word and nonword decisions. This task can be thought of as an analogue of face familiarity tasks only if the identical stimulus picture is used on successive trial blocks. This is the paradigm used in Experiment 1. However, in everyday face processing we are rarely, if ever, exposed to exactly the same facial stimulus. This begs the question as to what constitutes an instance in the face recognition domain and is the focus of Experiment 2. Together, in these experiments RT performance distributions were examined in which the same stimuli were repeated on each trial and this was compared with the more ecologically valid situation in which the pose and expression of an individual face varies from trial to trial.

\section{EXPERIMENT 1}

Ellis et al. (1996) differentiated two loci that mediate repetition priming in the recognition of familiar faces. The first involves the perceptual recognition of a face as familiar and is domain specific. That is, deciding that a face is familiar is primed only by the previous presentation of some representation of that face.

Experiment 1 employed the simplest possible variant of the tasks used in the previous research on differences between familiar- and unfamiliar-face processing (e.g., Bruce \& Valentine, 1985; Ellis et al., 1987). Familiarity judgments were made to the same photographs of famous and unfamiliar faces. The primary aim was to investigate the effects of repetition priming by examining the RT benefits that occur after more than one repetition of the photograph. This study extended the basic priming paradigm to allow examination of the benefits to performance through repeated presentation of the same photograph and also provided a direct-face processing analogy to Logan's (1988) experiments, in which the stimuli were words. 


\section{Method}

Subjects. Thirty psychology students from Lancaster University were the subjects. All had normal or corrected vision and had been exposed for a minimum of 5 years to the British media. They ranged in age from 19 to 32 years and were paid for their participation.

Stimuli and Materials. Forty-six monochrome images were "frame-grabbed" (using the QuickImage system) from videotapes of a range of television programs. The images that were selected ranged from three-quarter right, through full face, to three-quarter left poses and contained a variety of facial expressions. Twentythree of the images were of celebrities drawn from as wide a range of interests as possible. Each of these was then paired with an image of an unfamiliar face matched on age, pose, facial hair, and spectacle use. The selected images were then standardized by cropping the images to maximize the amount of facial information and to minimize the amount of background and clothing. The images were then standardized in size $(6.5 \times 4 \mathrm{~cm})$ and equated in brightness and contrast using Adobe Photoshop software and a Macintosh computer.

The stimuli were presented on Macintosh LCII computers with color monitors. These were placed at approximately eye level (i.e., the center of the screen was $35 \mathrm{~cm}$ above the height of the desk at which subjects were seated) and were situated behind a black screen positioned approximately $60 \mathrm{~cm}$ from the subject that allowed only the monitor to be viewed. The subjects made their responses by pressing one of two buttons on a button box placed on the desk in front of them. The buttons were connected to the computer, and each simulated a single keypress of a keyboard key. A filler task was also used between experimental blocks to ensure that the subjects had short breaks of approximately $3 \mathrm{~min}$. It required the subjects to make word/nonword judgments to lists of letter strings.

Experimental Design. The experimental design and stimulus presentation was handled by the SuperLab application for Macintosh computers. The subjects first viewed two screens of instructions before completing four practice trials, two of which presented images of celebrities and two of which presented images of unfamiliar persons. These were followed by a screen that listed the instructions for the experiment and that informed the subjects that they now had an opportunity to ask questions.

There followed an experimental block that consisted of 6 lead-in trials (the data from which did not enter into the analyses) and 40 experimental trials. Half of the trials presented images of celebrities and half presented images of unfamiliar faces. Both the leadin trials and the experimental trials were randomized before each presentation, and the subjects viewed the trail block eight times. Between experimental blocks, the subjects were required to complete one of the pages of the filler task word booklet.

The background color of the screen for each of the lead-in and experimental trials was pale blue, on which the word "ready" appeared in red letters approximately $1.5 \mathrm{~cm}$ tall. This was displayed for 2,000 msec in the center of the screen and was replaced after a 500 -msec interstimulus interval (ISI) with a central red dot, which was presented for $500 \mathrm{msec}$ and was again followed by a $500-\mathrm{msec}$ ISI followed by a stimulus face presented centrally for $2,500 \mathrm{msec}$. The subjects were then required to respond by pressing one of the two buttons. A further 1,000-msec ISI preceded the presentation of the "ready" signal, which indicated the start of the next trial sequence. After each block of trials, instructions to fill in one of the pages of the word booklet appeared.

Procedure. Each subject sat at a desk facing the monitor and was instructed to place the index finger of each hand on the two buttons and to locate the button box from a comfortable position. Each was then asked to read the instructions presented on the screen, which indicated that the experiment was designed to investigate how familiar and unfamiliar faces are processed and that a series of faces was to be presented. The subjects had to decide whether a particular face was one of a famous celebrity or of someone unknown at the start of the experiment and had to indicate their decisions by pressing the appropriate button. They were asked to make their decisions as quickly and as accurately as possible and to complete the practice trials. At the end of the practice trials, the experimenter indicated what the correct responses were and asked whether the subjects had any problems or questions. The experimenter then verbally repeated the instructions that the subjects should be as fast and as accurate as possible before the subjects started the experiment proper. Each subject then completed eight consecutive experimental blocks interspersed by their completion of one page of the filler task booklet.

For half of the subjects, pressing the right button indicated that the image was that of a celebrity, for the other half, the mapping was reversed.

\section{Results}

Two forms of analyses were done. First, analyses of variance (ANOVAs) were conducted on the RT data, since this has been the primary method used in previous face-priming research on differences between familiarand unfamiliar-face processing. Second, power curve parameters were fit to the data to examine the validity of the instance-based model and to allow comparisons between the forms of analyses presented in this study and in the series of studies following Logan (1988).

\section{ANOVAs of the RT Data}

For each subject, the RTs from the 20 famous and 20 unfamiliar faces as well as the errors were collected. The average error rate for famous faces was $1.99 \%$, and for unfamiliar faces, 3.19\%. Because so few errors occurred, no formal analyses were conducted. Trials on which errors were made were removed, and the mean correct RT and the standard deviation for each subject for each trial block for both stimulus classes were calculated. This generated a $2 \times 8$ within-subjects (type of face $\times$ experimental block) design, and the subsequent ANOVA revealed both main effects to be significant (see Table 1). Famous faces were recognized significantly faster than unfamiliar faces $\left[F(1,29)=57.15, M S_{\mathrm{e}}=127,555.07\right.$, $p<.001]$, and performance over the experimental blocks showed the expected practice-curve decrease $[F(7,203)=$ $\left.62.68, M S_{\mathrm{e}}=3,284.51, p<.001\right]$.

Table 1

The ANOVA-Based Mean Response Times (RT, in Milliseconds) and Mean Standard Deviations from Experiment 1 for Each of the Classes of Face and for Each Experimental Trial Block

\begin{tabular}{ccc}
\hline & \multicolumn{2}{c}{ Mean } \\
\cline { 2 - 3 } Condition & RT & $S D$ \\
\hline Type of Face & & \\
Famous & 595 & 104.1 \\
Unfamiliar & 673 & 116.1 \\
Block & & \\
1 & 761 & 137.1 \\
2 & 671 & 127.5 \\
3 & 640 & 108 \\
4 & 615 & 100.8 \\
5 & 602 & 98.9 \\
6 & 601 & 104.9 \\
7 & 589 & 96 \\
8 & 591 & 96.5 \\
\hline
\end{tabular}


The interaction between type of face and experimental block (see Figure 1) was also significant $[F(7,203)=$ $\left.11.78, M S_{\mathrm{e}}=1,248.77, p<.001\right]$. This occurred as a result of the RT's to familiar faces taking fewer trials to drop from their initial position to their asymptotic level-that is, they showed a much steeper rate of decrease than did unfamiliar faces. Exploration of this interaction involved conducting a number of comparisons. Since most of repetition priming experiments that use faces have involved only one repetition, the first analysis examined performance on the initial two trials. This indicated that the overall type of face $\times$ block interaction was not due to a differential reduction in RT for familiar and unfamiliar faces on the first two trials $\left[F(1,29)=0.007, M S_{\mathrm{e}}=0.11\right.$, $p=.993]$. Similar analyses on subsequent trial pairs indicated that the reduction in mean RT on Trials 2 and 3 for unfamiliar faces was $50.7 \mathrm{msec}$, which was significantly greater than the drop in RT for familiar faces, which was $36.4 \mathrm{msec}\left[F(1,29)=5.21, M S_{\mathrm{e}}=3,842.10\right.$, $p<.05]$. As will be seen, this is consistent with the power function fits which indicate that recognition of unfamiliar faces continues to improve over a number of trails, whereas recognition of familiar faces approaches asymptotic performance much more quickly.

A similar overall analysis was conducted on the standard deviations calculated from the RT data. For each subject, the standard deviation of the scores for famous and unfamiliar faces in each of the experimental blocks was calculated, producing a $2 \times 8$ (type of face $\times$ experimental block) within-subjects design. A subsequent ANOVA revealed both main effects to be significant (see Table 1). Standard deviations for famous faces were significantly lower than those for unfamiliar faces $[F(1,29)=$ $10.98, M S_{\mathrm{e}}=2,370.33, p<.0025$ ], and performance over the experimental blocks mirrored the practice-curve decrease seen for the mean RTs $\left[F(7,203)=9.212, M S_{\mathrm{e}}=\right.$ $1,528.74, p<.0001]$. The interaction, although of a sim-

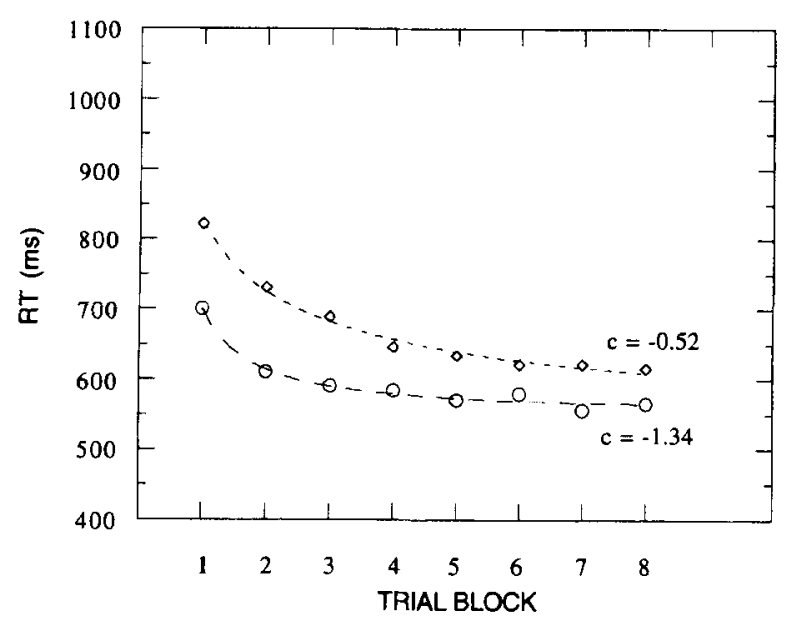

Figure 1. Power functions fit to the mean correct RT data (left) and the corresponding standard deviation data (right) obtained in Experiment 1 . The values of $c$ given refer to the learning rate parameter for the unfamiliar face data $(\diamond)$ and the familiar face data (O).



ilar shape to that observed for the mean RT data (see Figure 1), did not reach significance.

\section{Power Curve Parameter Estimation}

The instance theory detailed by Logan $(1988,1992)$ makes three strong predictions. First, the data from conditions in which subjects make the same decisions about the same stimuli in repeated blocks of trials should be well fit by power functions of the form

$$
\mathrm{RT}=a+b(\text { Trial Block })^{-c} .
$$

Second, the mean and standard deviation power functions of the data from each type of face should be well fit by power functions and should have the same $c$ parameter (Logan, 1988). Third, the quantiles of the RT data distributions should also be well fit by power functions and have common exponents.

The analysis strategy used to examine these predictions involved fitting power functions to various RT summaries. A number of different algorithms were employed, including the STEPIT algorithm (Chandler, 1965) used by Logan $(1988,1992)$, the Newton, the Quasi-Newton, the Steepest Descent algorithm (Raner, 1994), and the Levenberg-Marquard algorithm (Press, Flannery, Teukolosky, \& Vetterling, 1992). These all produced similar solutions. The prediction of common rate exponents was examined by constraining the $c$ parameter to be equal across functions while allowing the other parameters to vary freely, and by selecting the common exponent that minimized the error fit statistics for the functions under consideration. The constrained fits could then be compared with the unconstrained fits as a means of examining the validity of the instance theory predictions (Logan, 1988). The comparison between a constrained fit and the corresponding unconstrained fit is equivalent to the situation in which an additional independent variable is added to a regression equation. In such a situation it is pos- 
sible to test whether there has been a significant change in explained variance $\left(R^{2}\right)$ by evaluating the corresponding $t$ statistic.

The data for examining the predictions that are related to the distribution quantiles were prepared for this analysis by combining the individual subject's RTs to produce a group RT distribution calculated over five quantiles. Thus, for each subject, five quantiles (i.e., the quintiles in the 10th, 30th, 70th, and 90th percentiles) were calculated and averaged over subjects (see Ratcliff, 1979, for a full discussion of group RT distributions and quantile calculations). For completeness, the summary statistics for each block (collapsed over subject and stimulus) were also calculated. These yielded similar power functions, and so only the quintile data are presented.

Employing this strategy, power functions were fit to the overall mean RT and standard deviation data (see Figure 1). The estimated parameters and the measures of goodness of fit are presented in Table 2 . These clearly show that the data are well fit by power functions (alternative functions were also explored but in all cases power functions produced superior fits) and that the prediction, generated by Logan's (1988) instance theory, that the mean and the standard deviation functions should exhibit common exponents ( $c$ parameters) is supported for both famous and unfamiliar faces. These show different forms of processing for the famous-face functions, which show a rate of decline more than twice that for unfamiliar faces. Moreover, when the exponents for the mean and standard deviation functions were constrained to be equal and to minimize the error measures for each type of face, the fits were only marginally poorer than when the parameters were unconstrained. This conclusion is supported by the analysis of the changes in the values of $R^{2}$, which were found to be nonsignificant in all cases (see Table 2).

Table 2

Parameter Estimates From Fits of Power Functions

$\left[\mathrm{RT}=a+b\left(\right.\right.$ Block $\left.^{-c}\right]$ to Means and Standard Deviations of Response Times to the Famous and Unfamiliar Faces in Experiment 1

\begin{tabular}{|c|c|c|c|c|c|}
\hline Fit & $a$ & $b$ & $c$ & $R^{2}$ & RMSD \\
\hline \multicolumn{6}{|c|}{ Famous Face Mean RT $(t=0.91$, n.s. $)$} \\
\hline Unconstrained & 557 & 144 & -1.34 & .982 & 7.72 \\
\hline Constrained & 551 & 148 & -1.18 & .979 & 7.78 \\
\hline \multicolumn{6}{|c|}{ Famous Face $S D$ RT $(t=0.32$, n.s. $)$} \\
\hline Unconstrained & 77 & 59 & -0.90 & .905 & 6.40 \\
\hline Constrained & 83 & 54 & -1.18 & .903 & 6.48 \\
\hline \multicolumn{6}{|c|}{ Unfamiliar Face Mean RT ( $t=0.00$.n.s. $)$} \\
\hline Unconstrained & 500 & 324 & -0.52 & .991 & 8.26 \\
\hline Constrained & 483 & 340 & -0.48 & .991 & 8.34 \\
\hline \multicolumn{6}{|c|}{ Unfamiliar Face $S D$ RT $(t=0.31$, n.s. $)$} \\
\hline Unconstrained & 72 & 70 & -0.41 & .842 & 6.93 \\
\hline Constrained & 79 & 64 & -0.48 & .839 & 7.00 \\
\hline
\end{tabular}

Note-The significance of the decrease in $R^{2}$ due to constraining the $c$ parameter is given by the value of $t$, which was nonsignificant in all cases.
The power functions were also fit to the quantile data from the famous- and the unfamiliar-face data (see Figure 2). The estimated parameters and the goodness-of-fit measures to the quantile data from both famous and unfamiliar faces are presented in Table 3. As before, power functions fit the data from both famous and unfamiliar faces extremely well, with the exponents for the different quantile functions being similar within each type of face. The $c$ parameters of the individual quantile functions were also constrained to be equal to the value used to constrain the overall data for famous and unfamiliar faces, and again the decrease in $R^{2}$ was nonsignificant in all cases (see Table 3).

\section{DISCUSSION}

The ANOVA and curve-fitting results produced a consistent picture which confirms that both familiar and unfamiliar faces elicit RT performance curves that are well fit by power functions. Not only are the mean and standard deviation data well fit by power functions, but there is also strong support for the prediction that these and the quintile data have similar power function exponents. These data are in line with the predictions made by Logan's (1988) instance model. In contrast, current models of face processing that are based on FRUs, such as that of Bruce and Young (1986) or Burton et al.'s (1990) neural net implementation of McClelland and Rumelhart's (1981) interactive activation model, are unable to make quantitative RT predictions of this detail.

These models have particular difficulty in explaining the speedup in the processing of unfamiliar faces. Since these have no FRU, they should not elicit patterns of RT speedup similar to those shown by the processing of familiar faces. This finding clearly highlights one of the key gaps in structural-model accounts of face processingnamely, the processes by which FRUs are formed and how they interact with the processing of unfamiliar faces.

However, although the data from the present experiment indicate that the RT functions for the processing of familiar and unfamiliar faces are similarly shaped, these are not identical. The major difference between these functions lies in the first half of the RT curves. Familiar faces are initially processed more quickly but have an asymptote similar to that for unfamiliar faces. In fact, constraining the asymptote to be the same for familiar and unfamiliar faces makes little difference to the power function fits. However, the processing of familiar faces does exhibit a steeper learning rate (i.e., a larger $c$ parameter). Such a pattern is consistent with the findings in studies using similar paradigms with familiar and unfamiliar letter strings (Logan, 1988, 1990). This pattern indicates that repeated exposure is sufficient, in the long run, for unfamiliar faces to be processed like familiar faces and argues against the notion of a processing change that is dependent on the formation of a new structure such as an FRU. The initial differences in the shapes of the RT functions could be explained by the fact that familiar faces already 

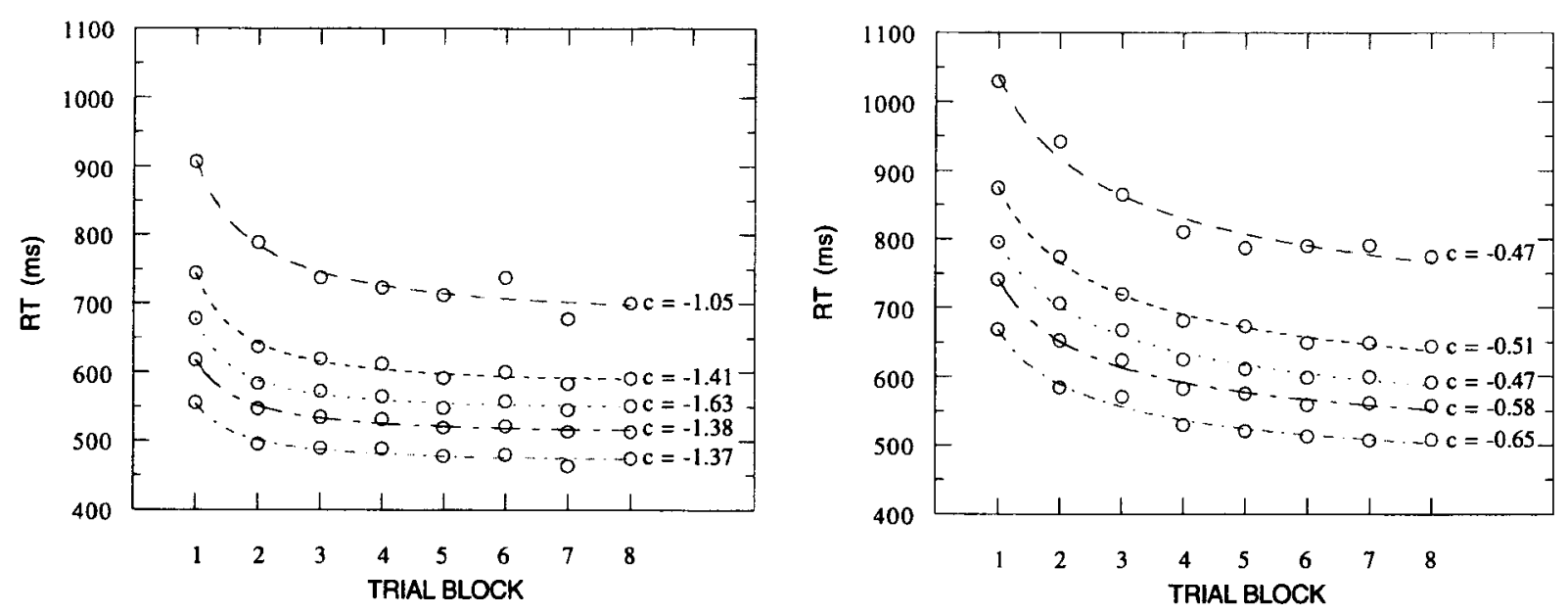

Figure 2. Power functions fit to the quantiles of $R T$ data for correct responses to familiar faces (left) and unfamiliar faces (right) from Experiment 1 . The values of $c$ given refer to the learning rate parameter.

have existing instances to assist in their processing whereas processing of unfamiliar faces must create a minimal number of new instances early in the experiment.

What complicates this simple explanation is that the word studies of Logan and the present study used exactly the same stimuli on each trial. Although this is a legitimate tactic in the study of word recognition, it is far from ecologically valid in the face-processing domain, where seeing exactly the same stimulus twice is the exception.

Table 3

Parameter Estimates from Unconstrained and Constrained Fits of Power Functions [RT $=a+b$ (Block) ${ }^{-c} \mid$ to the Five Quantiles of the Response Time (RT) Distributions of the Famous and the Unfamiliar Faces Used in Experiment 1

\begin{tabular}{lccccccr}
\hline Type of Face & Quantile & $a$ & $b$ & $c$ & $R^{2}$ & $t$ & RMSD \\
\hline \multirow{6}{*}{ Famous } & 1 & 671 & 236 & -1.05 & 0.954 & & 18.28 \\
& 2 & 581 & 163 & -1.41 & 0.986 & & 7.28 \\
& 3 & 545 & 133 & -1.63 & 0.986 & & 6.19 \\
& 4 & 510 & 108 & -1.38 & 0.990 & & 4.15 \\
Unfamiliar & 5 & 468 & 86 & -1.37 & 0.958 & & 6.79 \\
& 1 & 592 & 445 & -0.45 & 0.971 & & 18.48 \\
& 2 & 519 & 357 & -0.53 & 0.994 & & 7.32 \\
& 3 & 468 & 329 & -0.49 & 0.991 & & 7.78 \\
& 4 & 475 & 267 & -0.60 & 0.991 & & 7.74 \\
& 5 & 438 & 230 & -0.61 & 0.985 & & 8.10 \\
Famous & & Constrained Fits & & & \\
& 1 & 680 & 229 & -1.18 & 0.953 & 0.33 & 18.47 \\
& 2 & 572 & 169 & -1.18 & 0.984 & 0.85 & 7.91 \\
& 3 & 534 & 141 & -1.18 & 0.977 & 1.79 & 7.86 \\
& 4 & 504 & 112 & -1.18 & 0.988 & 1.00 & 4.53 \\
Unfamiliar & 5 & 464 & 89 & -1.18 & 0.956 & 0.49 & 6.92 \\
& 1 & 610 & 427 & -0.48 & 0.971 & 0.00 & 18.51 \\
& 2 & 498 & 377 & -0.48 & 0.994 & 0.00 & 7.46 \\
& 3 & 463 & 334 & -0.48 & 0.991 & 0.00 & 7.78 \\
& 4 & 439 & 301 & -0.48 & 0.988 & 1.00 & 8.21 \\
& 5 & 404 & 261 & -0.48 & 0.984 & 0.58 & 8.48 \\
\hline
\end{tabular}

Note-The significance of the decrease in $R^{2}$ due to constraining the $c$ parameter is given by the value of $t$ which was nonsignificant in all cases.
Changes in pose, lighting, expression, hairstyle, and age mean that a range of discrepancies is possible between two exposures. Would such different stimuli be considered instances? If not, the RT functions observed in this experiment could not be repeated, and the usefulness of Logan's instance model would be severely limited in its application.

\section{EXPERIMENT 2}

As has been frequently pointed out (e.g., Hay \& Young, 1982; Hay, Young, \& Ellis, 1986), recognizing the same photograph may not employ exactly the same processes that are used in recognizing individuals in real life situations, in which the stimulus involved is unlikely ever to be exactly the same as the one encountered previously. In fact, face recognition may best be considered as a visual categorization task in which a new stimulus (e.g., a new exposure to Madonna) is assigned to the visual category of Madonna's face.

Thus, in an attempt to be more ecologically valid, different poses were employed in each of the eight trial blocks in Experiment 2.

\section{Method}

Subjects. Thirty psychology students from Lancaster University were the subjects. All had normal or corrected vision and had been exposed for a minimum of 5 years to the British media. They ranged in age from 19 to 30 years and were paid for their participation.

Stimuli and Materials. Video clips of a range of celebrities were collected from TV productions. Each was around $2 \mathrm{~min}$ in duration and contained a range of head movements and expressions. From these 23 celebrities were drawn a sample as wide in range of interests as possible. Similarly, clips of unfamiliar faces were collected from German and Dutch TV programs and films in an attempt to equate the quality and range of faces. Twenty-three of these were selected to match the chosen celebrities on gender, age, facial hair, and spectacle use.

The video clips yielded eight monochrome images that were "frame-grabbed" using the QuickImage system. The images se- 
lected for each individual face ranged from three-quarter right, through full face, to three-quarter left poses, and contained a variety of facial expressions. The selected images were then standardized by first cropping the image to maximize the amount of facial information while minimizing the amount of background and clothing. Images were then standardized in size $(6.5 \times 4 \mathrm{~cm})$ and equated in brightness and contrast using Adobe Photoshop software on a Macintosh computer.

Procedure. All other aspects of the procedure were the same as in Experiment 1.

\section{Results}

The initial analyses were done in the same form as those detailed in Experiment 1.

ANOVAs of the RT Data. For each subject, the RTs from the 20 famous and the 20 unfamiliar faces and the errors were collected. The error rate for famous faces was $2.35 \%$, and for unfamiliar faces, $4.13 \%$. Trials on which an error was made were removed, and the mean correct RT and the standard deviation for each subject for each trial block for both stimulus classes were calculated.

This yielded mean RT data in a $2 \times 8$ (type of face $\times$ experimental block) within-subjects design. A subsequent ANOVA revealed that both main effects were significant (see Table 4). Famous faces were recognized significantly faster than unfamiliar faces $[F(1,29)=79.74$, $\left.M S_{\mathrm{e}}=11,587.50, p<.0001\right]$, and performance over the experimental blocks showed the usual practice-curve decrease $\left[F(7,203)=76.44, M S_{\mathrm{e}}=2,237.46, p<.0001\right]$.

In addition, the interaction between type of face and experimental block (see Figure 3) was also significant. As in Experiment 1, there was a much steeper rate of decrease for famous faces than for unfamiliar faces $[F(7,203)=$ $\left.4.42, M S_{\mathrm{e}}=868.57, p<.0001\right]$. A similar analysis was conducted on the standard deviation data. For each subject, the standard deviation of the scores for famous and unfamiliar faces in each of the experimental blocks was calculated, producing a $2 \times 8$ (type of face $\times$ experimental block) within-subjects design. A subsequent ANOVA revealed both main effects to be significant (see Table 4). Standard deviations for famous faces were significantly lower than those for unfamiliar faces $[F(1,29)=$

Table 4

The ANOVA-Based Mean Response Times (RT, in Milliseconds) and Mean Standard Deviations from Experiment 2 for Each of the Classes of Face and for Each Experimental Trial Block

\begin{tabular}{lrr}
\hline & \multicolumn{2}{c}{ Mean } \\
\cline { 3 - 3 } Condition & RT & $S D$ \\
\hline Type of Face & & \\
Famous & 592 & 105.1 \\
Unfamiliar & 680 & 112.4 \\
Block & & \\
1 & 754 & 135.4 \\
2 & 665 & 111.7 \\
3 & 643 & 113.9 \\
4 & 622 & 107.8 \\
5 & 611 & 98.3 \\
6 & 605 & 107.2 \\
7 & 595 & 99.5 \\
8 & 595 & 96.4 \\
\hline
\end{tabular}

$\left.7.52, M S_{\mathrm{e}}=855.049, p<.05\right]$, and performance over the experimental blocks mirrored the practice-curve decrease seen for the mean RTs $\left[F(7,203)=6.26, M S_{\mathrm{e}}=424.764\right.$, $p<.001]$. The interaction, although of a similar shape to that observed for the mean RT (see Figure 3), did not reach significance.

Power curve parameter estimation. As before, power functions produced better fits than did other similarly shaped functions, and so only the power fit data are presented. The data were prepared for this analysis by combining the individual subjects' RTs to produce a group RT distribution calculated over five quantiles.

Power functions were fit to the overall mean RT and standard deviation data (see Figure 3), and the estimated parameters and the measures of goodness of fit are presented in Table 4 . These clearly show that the data are well fit by power functions and that the prediction generated by Logan's (1988) instance theory of the mean and the standard deviation functions exhibiting common exponents ( $c$ parameters) is supported for both famous and unfamiliar faces. These show different forms of processing, with the famous-face functions showing a rate of decline more than twice that shown for unfamiliar faces. Moreover, when the exponents for means and standard deviations were constrained to be equal and to minimize the error measure for each type of face, the marginal decrease in $R^{2}$ between unconstrained and constrained fits was found to be nonsignificant, all values of $t$ being less than one (see Table 5).

Power functions were also fit to the quantile data from famous- and unfamiliar-face data (see Figure 4). The estimated parameters and the goodness-of-fit measures to the quantile data from both famous and unfamiliar faces are presented in Table 3. As before, power functions fit the data from both famous and unfamiliar faces extremely well, with the exponents for the different quantile functions being similar within each type for face. The $c$ parameter of the individual quantile functions was again constrained to be equal to that used when constraining the overall data for famous and unfamiliar faces (see Table 5). As before, the constrained functions produced parameters and fits very similar to those generated by the unconstrained fits. In all cases, the change in $R^{2}$ between constrained and unconstrained fits was found not to differ significantly (see Table 6).

\section{Comparisons of Experiments 1 and 2}

The analyses so far have indicated a good fit between the predictions made by Logan's instance model and the present data. Additional analyses were conducted to further determine whether changes in response performance were dependent on a move from identical stimuli presented on each trial to stimuli that changed in pose and expression from trial to trial. Of particular importance to the question of what constitutes an instance are the interactions, for each type of face, between type of pose (i.e., fixed pose in Experiment 1 and varied pose in Experiment 2) and performance over trials. Planned compar- 

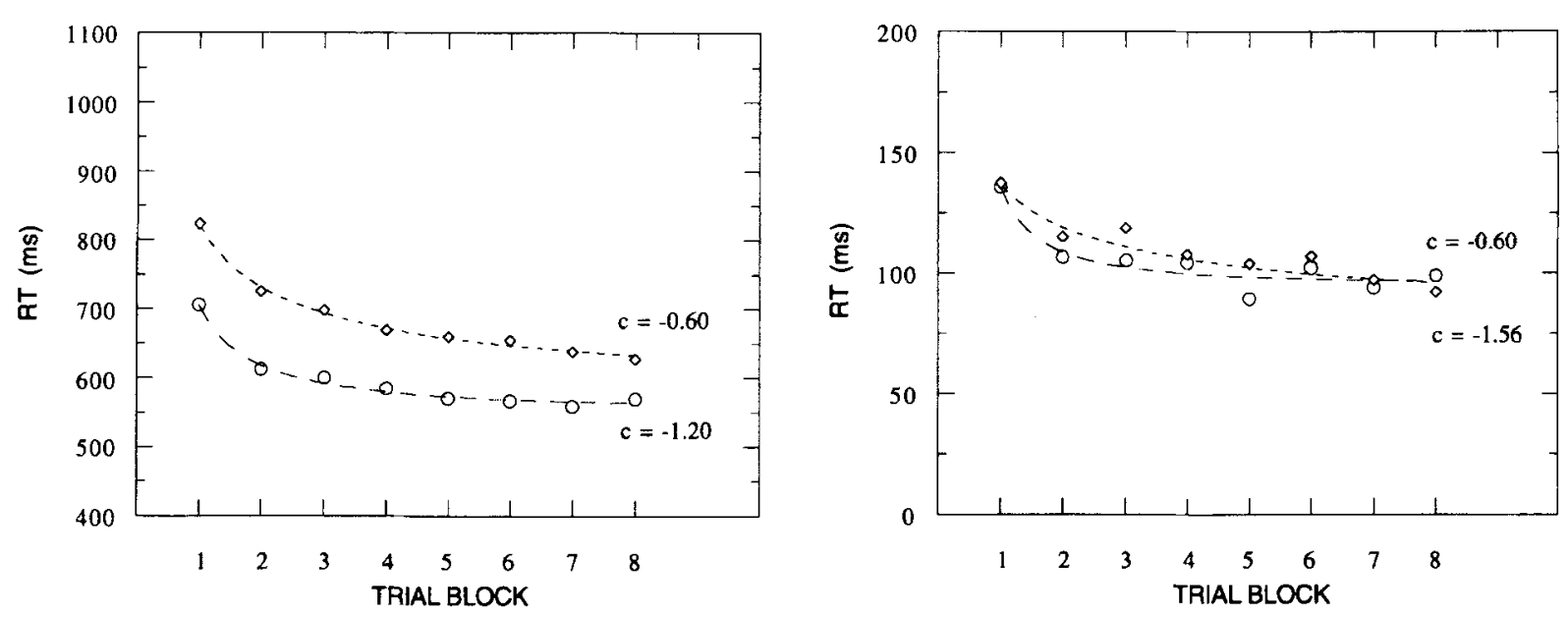

Figure 3. Power functions fit to the mean correct RT data (left) and the corresponding standard deviation data (right) obtained in Experiment 2. The values of $c$ given refer to the learning rate parameter for the unfamiliar face data $(\diamond)$ and the familiar face data $(0)$.

isons revealed that these interactions were not significant for familiar faces $\left[F(7,406)=0.59, M S_{\mathrm{e}}=1,373.15\right.$, $p>.05]$ or for unfamiliar faces $\left[F(7,406)=1.44, M S_{\mathrm{e}}=\right.$ $244.50, p>.05]$. Similarly, no significant differences involving pose were found in analyses of the standard deviation data. The similarity between the power curves across experiments can be seen by comparing the data plotted in Figures 1 and 3 and the curve fits displayed in Tables 2 and 5 . To assess the consistency of this pattern, power curves were fit to each subject's familiar- and unfamiliar-face data in both Experiment 1 and Experiment 2 . The increased noise inherent in the individual data meant that it was impossible to fit power functions in all cases that had positive values for the asymptotic (i.e., the $a$ ) parameter. Negative values are psychologi-

Table 5

Parameter Estimates From Fits of Power Functions

[RT $=a+b$ (Block) $\left.^{-c}\right]$ to Means and Standard Deviations of Response Times to the Famous and Unfamiliar Faces in Experiment 2

\begin{tabular}{|c|c|c|c|c|c|}
\hline Fit & $a$ & $b$ & $c$ & $R^{2}$ & RMSL \\
\hline \multicolumn{6}{|c|}{ Famous Face Mean RT $(t=0.61$, n.s. $)$} \\
\hline $\begin{array}{l}\text { Unconstrained } \\
\text { Constrained }\end{array}$ & $\begin{array}{l}551 \\
555\end{array}$ & $\begin{array}{l}154 \\
151\end{array}$ & $\begin{array}{r}-1.20 \\
1.31\end{array}$ & $\begin{array}{l}0.987 \\
0.986\end{array}$ & $\begin{array}{l}6.43 \\
6.58\end{array}$ \\
\hline \multicolumn{6}{|c|}{ Famous Face $S D$ RT $(t=0.30$, n.s. $)$} \\
\hline $\begin{array}{l}\text { Unconstrained } \\
\text { Constrained }\end{array}$ & $\begin{array}{l}95 \\
93\end{array}$ & $\begin{array}{l}40 \\
42\end{array}$ & $\begin{array}{l}-1.56 \\
-1.31\end{array}$ & $\begin{array}{l}0.887 \\
0.885\end{array}$ & $\begin{array}{l}5.53 \\
5.60\end{array}$ \\
\hline \multicolumn{6}{|c|}{ Unfamiliar Face Mean RT $(t=0.00$, .n.s. $)$} \\
\hline $\begin{array}{l}\text { Unconstrained } \\
\text { Constrained }\end{array}$ & $\begin{array}{l}560 \\
556\end{array}$ & $\begin{array}{l}262 \\
265\end{array}$ & $\begin{array}{l}-0.61 \\
-0.61\end{array}$ & $\begin{array}{l}0.994 \\
0.994\end{array}$ & $\begin{array}{l}5.76 \\
5.76\end{array}$ \\
\hline \multicolumn{6}{|c|}{ Unfamiliar Face $S D$ RT $(t=0.00$, n.s. $)$} \\
\hline $\begin{array}{l}\text { Unconstrained } \\
\text { Constrained }\end{array}$ & $\begin{array}{l}85 \\
85\end{array}$ & $\begin{array}{l}53 \\
53\end{array}$ & $\begin{array}{l}-0.60 \\
-0.60\end{array}$ & $\begin{array}{l}0.878 \\
0.878\end{array}$ & $\begin{array}{l}5.81 \\
5.81\end{array}$ \\
\hline
\end{tabular}

Note-The significance of the decrease in $R^{2}$ due to constraining the $c$ parameter is given by the value of $t$, which was nonsignificant in all cases. cally impossible, which implies that performance speeds up to the extent of producing negative RTs. This pattern was observed in only 13 cases ( 9 from Experiment 1 and 4 from Experiment 2), and the data from these subjects were removed from the following analyses. Separate 2 $\times 2$ (type of pose $\times$ type of face) ANOVAs were conducted for each of the three estimated parameters. Investigation of the asymptotic parameters (the $a$ parameters) revealed no significant differences between type of pose or type of familiar face, nor any interaction between these factors. For the $b$ parameter-the measure of the difference between initial performance and asymptotic performance - the analysis indicated a significant main effect only of familiarity, with the $b$ parameter being significantly less for familiar faces (194 msec) than for unfamiliar faces $(317 \mathrm{msec})\left[F(1,45)=22.05, M S_{\mathrm{e}}=16,115.1, p<.001\right]$. Similarly, the analysis of the $c$ parameter - the index of the rate of learning-also revealed a significant effect only of familiarity $\left[F(1,45)=5.56, M S_{\mathrm{e}}=54.18, p<.05\right]$, which confirms that this was significantly greater for familiar $(-3.82)$ than for unfamiliar $(-0.95)$ faces.

\section{Discussion}

As before, the data were well fit by power functions, and both the standard deviation and the mean RT curves for familiar and unfamiliar faces exhibited functions with learning indices similar to those observed in Experiment 1 . Because the quantile data were also well fit by power curves with similar indices, the data again offer strong support for the predictions generated by Logan's instance model. In Experiment 2, the varying of poses from trial-to-trial produced no observable differences from the functions and parameters observed in Experiment 1 . This confirms the flexibility of Logan's model and demonstrates that it is not restricted to situations in which the stimulus-response instance is identical on consecutive trials. 

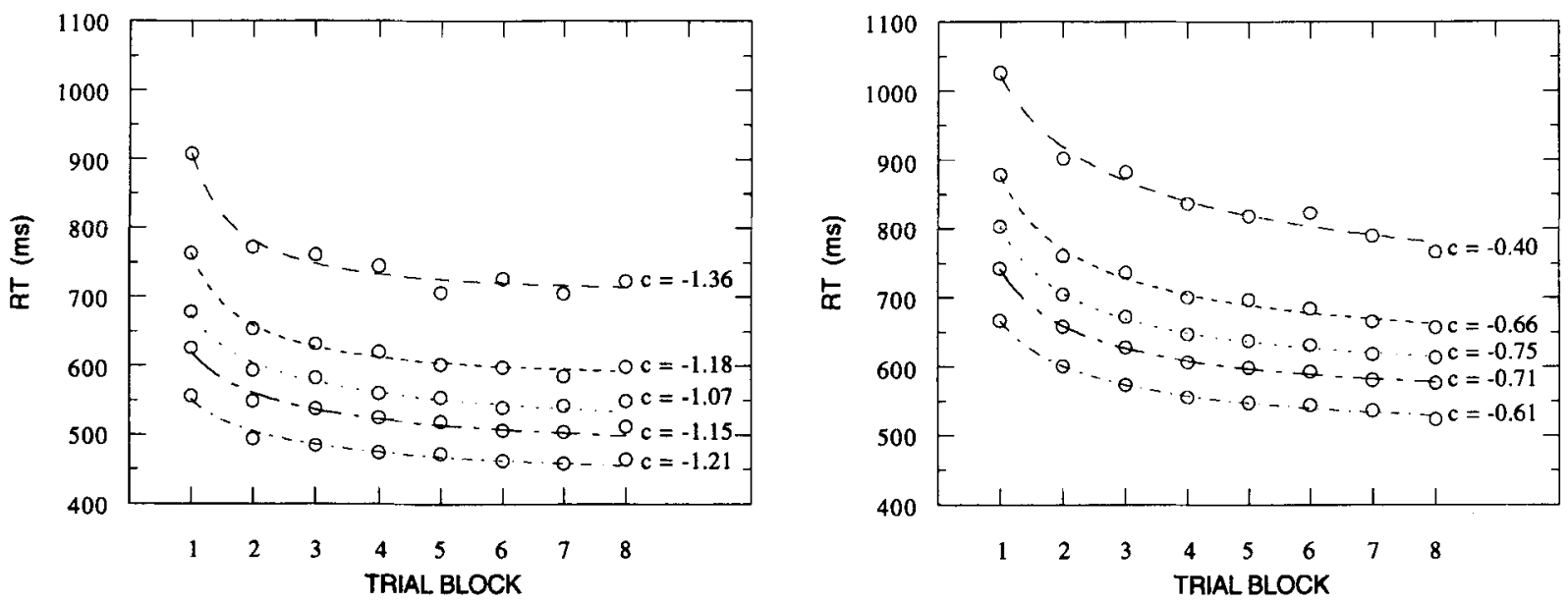

Figure 4. Power functions fit to the quantiles of $R T$ data for correct responses to familiar faces (left) and unfamiliar faces (right) from Experiment 2. The values of $c$ given refer to the learning rate parameter.

As before, processing of familiar faces yielded functions that differed from those obtained for the processing of unfamiliar faces. The differences were restricted to the parameters that measured early RT performance and not the level of the asymptote. The lower $b$ parameter was a result of the familiar-face function's having a lower performance on Trial 1, relative to the same asymptotic level that the $c$ parameter indicated was reached in fewer trials for familiar faces than for unfamiliar faces.

Table 6

Parameter Estimates From Unconstrained and Constrained Fits of Power Functions [RT $=a+b\left(\right.$ Block) ${ }^{-c}$ ] to the Five Quantiles of the Response Time Distributions of the Famous and the Unfamiliar Faces Used in Experiment 2

\begin{tabular}{lccccccr}
\hline Type of Face & Quantile & $a$ & $b$ & $c$ & $R^{2}$ & $t$ & RMSD \\
\hline \multirow{6}{*}{ Famous } & 1 & 702 & 204 & -1.36 & 0.965 & & 14.51 \\
& 2 & 576 & 186 & -1.18 & 0.990 & & 6.95 \\
& 3 & 526 & 152 & -1.07 & 0.983 & & 7.09 \\
& 4 & 495 & 129 & -1.15 & 0.986 & & 5.62 \\
Unfamiliar & 5 & 454 & 101 & -1.21 & 0.988 & & 4.17 \\
& 1 & 594 & 428 & -0.4 & 0.979 & & 14.11 \\
& 2 & 590 & 286 & -0.66 & 0.991 & & 8.06 \\
& 3 & 565 & 239 & -0.75 & 0.998 & & 3.00 \\
& 4 & 529 & 213 & -0.71 & 0.998 & & 2.62 \\
& 5 & 475 & 192 & -0.61 & 0.996 & & 3.50 \\
& & Constrained Fits & & & \\
& 1 & 700 & 205 & -1.31 & 0.965 & 0.00 & 14.54 \\
& 2 & 582 & 182 & -1.31 & 0.989 & 0.71 & 7.22 \\
& 3 & 536 & 144 & -1.31 & 0.980 & 0.94 & 7.72 \\
& 4 & 501 & 125 & -1.31 & 0.984 & 0.85 & 5.88 \\
Unfamiliar & 5 & 456 & 100 & -1.31 & 0.987 & 0.65 & 4.25 \\
& 1 & 690 & 338 & -0.6 & 0.976 & 0.85 & 15.14 \\
& 2 & 575 & 300 & -0.6 & 0.991 & 0.00 & 8.20 \\
& 3 & 536 & 264 & -0.6 & 0.997 & 1.58 & 4.34 \\
& 4 & 510 & 230 & -0.6 & 0.997 & 1.58 & 3.30 \\
& 5 & 474 & 193 & -0.6 & 0.996 & 0.00 & 3.51 \\
\hline
\end{tabular}

Note-The significance of the decrease in $R^{2}$ due to constraining the $c$ parameter is given by the value of $t$ which was nonsignificant in all cases.

\section{GENERAL DISCUSSION}

The main objective in this paper has been to rigorously examine the predicted power function speedup of RT that occurs in face-repetition priming. The findings presented here appear to offer clear support for the predictions made by Logan's instance model. Specifically, these are the following: (1) Speed-up in RT performance takes the form of a power function. This was observed in Experiments 1 and 2 for both familiar and unfamiliar faces. (2) The learning-rate parameter for the power functions fitted to the mean RT and the standard deviation of the RTs in each trial block were the same. The constrained fits support this position for RTs to both familiar and unfamiliar faces. And (3) the different quantiles of the RT distributions also share the same learning-rate parameter. Again the evidence from both experiments supports this prediction.

The present data pose a number of significant problems for structural accounts of face processing. Perhaps the most obvious is that these accounts can offer no predictions as to what RT performance in repetition priming tasks will be. Face recognition units (FRUs), like logogens, are black box constructs containing both the internal representation and the process by which it is matched with the incoming visual stimulus. The lack of specification makes these accounts useful descriptive devices but, consequentially, they have a low predictive power. The lowering of a threshold is seen as correlating with a reduction in $\mathrm{RT}$, but it remains to be seen how this mechanism can be modified to account for the power function speedup in the various measures of RT performance that have been demonstrated here. Structural accounts are also limited in that they deal only with preformed units, and they suggest that the processing of unfamiliar faces, which have no associated unit, should show no RT performance decrease with repetition. As in the Bentin and Moscovitch (1988) study, the present data revealed "priming 
like" behavior for the processing of unfamiliar faces. This was true even for the situation in which different poses of the same unfamiliar face were seen on different trials. Thus, the contention that face repetition priming effects occur only within the part of the system that handles familiar face recognition (Ellis et al., 1996; Ellis et al., 1990) has little support and is further weakened by the demonstration by Hay (1999) of repetition priming effects in an expression judgment task. It was revealed in Hay that "expression" priming for familiar and for unfamiliar faces were similar. More interestingly, Logan's instance theory was used to predict the conditions under which such effects could be obtained while having the results match the predictions.

Some integration of the FRU and instance model arguments may be possible. For example, the Burton et al. (1990) models are based on the interactive activation network suggested by McClelland and Rumelhart (1981). More recent and comprehensive versions of this model seek to explain the development of what appear to be abstractive word and concept units that result from the storage of all instances of the word or concept (McClelland \& Rumelhart, 1985). Models such as these respond strongly to prototypical patterns and also to recent instances in the training set.

The data from the present two experiments suggest various problems for Logan's instance model. Although the processing of both familiar and unfamiliar faces exhibits power function speedup, the consistent finding is that the shape of functions differ. Familiar faces are initially processed faster and have a greater learning-rate parameter. The initial fast processing of familiar faces is a common finding in face decision tasks (Bruce \& Valentine, 1985; Ellis et al., 1987; Hay et al., 1986; Young et al., 1986a). Both structural and instance theories can explain this phenomenon. The FRU model suggests that because unfamiliar faces have not previously been seen and the task requires a judgment of familiarity, deciding that a face is unfamiliar is the default option if a face is not considered familiar. In Logan's instance model, this is also a possibility that results from different algorithmic processes for familiar and unfamiliar faces.

The difference in the learning rates for familiar and unfamiliar faces could also be interpreted as evidence of a qualitatively different underlying processing mechanism, however, and the instance model offers two possibilities to explain the differential speedup. The first is related to one of the simplifying assumptions used by Logan (1988). Specifically, the algorithmic processing remains unchanged with practice. As Logan (1988) pointed out, this is unlikely to be true in general, and in his personal communication in response to Kirsner and Speelman (1996), he indicated how this model could be modified to support practice effects and the additive relationship that was observed between repetition priming and practice. Another possible explanation exists that is related to the question of what constitutes an instance and which instances enter the race. For familiar-face processing, it is possible that a number of preexperimental instances already exist. It could be that after the first trial, rather than only one "familiar face decision" instance being available, a number are sufficiently useful and enter the race. If the system is flexible enough, the number of $a p$ propriate instances might increase in subsequent trials until all available "familiar face decision" instances were employed. This implies that the processing of familiar faces is a function not of the number of experimental trials but of the number of instances in the race on any experimental trial. Unfortunately a host of possible instance values that increase over trials exist, all of which can be fit by power functions. However, it is interesting to note that one of those that produced a particularly good fit had power function parameters very similar to those found here for unfamiliar faces. This opens up the possibility that a single process underlies the repetition priming effects that were demonstrated here for both familiar and unfamiliar faces.

Although it is possible to adapt the basic instance model to account for the observed differences in familiar and unfamiliar function shape, there is evidence from both psychological and computational approaches to face recognition that is directly relevant to this discussion. These approaches concentrate exclusively on how suitable internal representations are derived from differing visual exemplars. The psychological studies, in which the rotational angle of the head is varied between initial presentation and recognition (usually some combination of frontal three-quarter and profile), support the broad conclusion that recognition performance varies with face familiarity. Processing of familiar faces tends to be insensitive to rotational transformation, whereas unfamiliar face recognition performance tends to decrease with rotational transformation (Bruce, 1982; Bruce, Valentine, \& Baddeley, 1987; Krouse, 1981). These differences in performance are frequently interpreted as being indicative of a qualitative difference in the nature of the internal representations formed for familiar and unfamiliar faces (Valentin, Abdi, \& Edelman, 1997). Such interpretations could be employed to explain the difference in the power functions observed here. The fact that the threequarter view of unfamiliar faces leads to better recognition performance than do other views is interpreted as being suggestive of a system with multiple view-dependent representations (Valentin et al., 1997). There seem to be two ways of implementing such multiple view systems; those systems that store sufficient instances to allow any novel view to be close to one of the image sets are variants of the Logan model in which all instances are stored, or are what Moses, Ullman, and Edelman (1996) called the interdependent approach. In this type of system, only a small number of specific orientations are stored and used to extract the three-dimensional information (e.g., Bülthoff, Edelman, \& Tarr, 1995; Edelman, 1995). In their simulations, Valentin et al. (1997) demonstrated that a system that stored only two views (frontal and profile) was sufficient to accurately identify $9 \%$ of multiple-pose 
face views. Such a system has a degree of neurophysiological validity, as has been shown by the single-cell recording of activity in the temporal cortex of monkeys presented with faces: Cells were found to have statistical preferences for these views (Perret, Heitanen, Oram, \& Benson, 1992). Unfortunately, these systems currently provide only accuracy data, whereas the Logan instance models provide only RT predictions. It remains to be seen whether systems based on the interdependent approach can reproduce repetition priming phenomena in general and RT power functions of the form reported here. Particularly important is the power function speedup associated with unfamiliar faces, which is related to recognition performance while new internal representations are being created and developed. The Logan model is especially suited to this incremental process.

However, it is unlikely that the link between the power function speedup of RT and Logan's instance theory is unique. Van Zandt and Ratcliff (1995) investigated a range of statistical architectures that mimic existing cognitive models. They showed that a mixture of gamma functions can produce RT data that have the characteristics predicted by Logan's model. Van Zandt and Ratcliff added, however, that there was no theoretical basis behind their use of gamma functions; they merely used these to demonstrate that alternatives exist. This is in contrast to Logan's instance theory, which is based on a number of explicit assumptions that predict distributional changes of the type observed in the present experiments.

There may also be a problem with the mathematical underpinnings of the instance theory, as is indicated by the interchange between Colonius (1995) and Logan (1995). Although both agree that the development of automaticity is well characterized by a race between instances, there appears to be a problem concerning the conditions under which it is justified to choose the Weibull distribution as the underlying parent distribution for the minima of the RTs. It appears that the argument used by Logan for choosing this function has at least one error, and Colonius suggested an alternative argument to support the choice of the Weibull distribution based on Huang's (1989) theorem, which proves that the sequence of means of minima uniquely determines the distribution of the minima. For example, if the mean RTs conform to a power function, the whole distribution of RTs is constrained to be this shape and the distributional indices such as the standard deviations and the quantiles will also exhibit this shape. Thus, this proof of the instance theory implies that the means constrain the shape of the distribution, which is neither the general case (Townsend, 1990), nor a property of alternative psychological theories (Compton \& Logan, 1991; Morton, 1979).

In his reply to Colonius, Logan (1995) suggested that before power function speedup in RT distributions is viewed as a cornerstone of the instance theory, he would like to see more evidence of its robustness and generality. The data presented here provide confirmation that the RT distributions from another psychological domain (i.e., face repetition priming) exhibit power function speedup. The challenge facing proponents of alternative approaches is clear. Can their approaches be modified and/or be better specified to that they encompass the present results, or is the instance theory the only existing viable model?

\section{REFERENCES}

Baddeley, A. D. (1982). Domains of recollection. Psychological Review, 89, 708-729.

Bentin, S., \& Moscovitch, M. (1988) The time course of repetition priming effects for words and unfamiliar faces. Journal of Experimental Psychology: General, 117, 148-160.

BRUCE, V. (1982). Changing faces: Visual and non-visual coding processes in face recognition. British Journal of Psychology, 73, 105 116.

Bruce, V., \& VAlentine, T. (1985). Identifying priming in the recognition of familiar faces. British Journal of Psychology, 76, 373-383.

Bruce, V., Valentine, T. \& Baddeley, A. (1987). The basis of the $3 / 4$ advantage in face recognition. Applied Cognitive Psychology, 1, 109. 120.

BRUCE, V., \& Young, A. W. (1986). Understanding face recognition. British Journal of Psychology, 77, 305-327.

Bülthoff, H. H., Edelman, S., \& TARR, M. J. (1995). How are threedimensional objects represented in the brain? Cerebral Cortex, 3 247-260.

Burton, A. M., Bruce, V., \& Johnston, R. A. (1990). Understanding face recognition with an interactive activation model. British Journal of Psychology, 81, 361-380.

CHANDLER, P. J. (1965). Subroutine STEPIT: An Algorithm that finds the values of the parameters which minimize a given continuous function [Computer program]. Bloomington: Indiana University, Quantum Chemistry Program Exchange.

Colonius, H. (1995). The instance theory of automaticity-why the Weibull? Psychological Review, 102, 744-750.

Compton, B. J., \& LOGAN, G. D. (1991). The transition from algorithm to retrieval in memory-based theories of automaticity. Memory \& Cognition, 19, 151-158.

Edelman, S. (1995). Representation, similarity and the chorus of prototypes. Minds \& Machines, 5, 45-68

Ellis, A. W., Flude, B., Bruce, V., \& Burton, A. M. (1996). Two loci of repetition of priming of familiar faces. Journal of Experimental Psychology: Learning, Memory, \& Cognition, 22, 295-308.

Ellis, A. W., Young, A. W., \& Flude, B. (1990). Repetition priming and face processing: Priming occurs within the system that responds to the identity of a face. Quarterly Journal of Experimental Psychol ogy, 42A, 495-512.

Ellis, A. W., Young, A. W., Flude, B., \& HaY, D. C. (1987). Repetition Priming of face recognition. Quarterly Journal of Experimental Psychology, 39A, 193-210.

HAY, D. C. (1999). Repetition priming of face gender judgments: An instance based explanation. Current Psychology, 18, 140-149.

HAY, D. C., \& Young, A. W. (1982). The human face. In A. W. Ellis (Ed.), Normality and Pathology in Cognitive Functions. New York: Academic Press.

HAY, D. C., YounG, A. W., \& ElLLIS, A. W. (1986). What happens when a face rings a bell: The automatic processing of famous faces. In H. D. Ellis, M. A. Jeeves, F. Newcombe, \& A. W. Young (Eds.), Aspects of Face Processing. Proceedings of the NATO Advanced Research Workshop (pp. 136-147). Dordrecht: Martinus Nijhoff.

HuAnG, J. S. (1989). Moment problem of order statistics: A review. International Statistical Review, 57, 59-66.

JАCOBY, L. L. (1983). Perceptual enhancement: Persistent effects of an experience. Journal of Experimental Psychology: Learning. Memory. \& Cognition, 9, 21-38.

JACOBY, L. L., \& BROOKS, L. R. (1984). Nonanalytic cognition: Mem- 
ory, perception and conceptual learning. In G. H. Bower (Ed.), The psychology of learning and motivation (Vol. 18, pp. 1-47). New York: Academic Press.

Kirsner, K., \& Speelman, C. (1996). Skill acquisition and repetition priming: One principle, many processes? Journal of Experimental Psychology: Learning, Memory, \& Cognition, 22, 563-575.

KrousE, F. (1981). Effects of pose, pose change, and delay on face recognition performance. Journal of Applied Psychology, 66, 651-654.

LOGAN, G. D. (1988). Towards an instance theory of automatization. Psychological Review, 95, 492-527.

LoGAN, G. D. (1990). Repetition priming and automaticity: Common underlying mechanisms? Cognitive Psychology, 22, 1-35.

LOGAN, G. D. (1992). Shapes of reaction-time distributions and shapes of learning curves: A test of the instance theory of automaticity. Journal of Experimental Psychology: Learning, Memory, \& Cognition, 18, 883-914.

LoGAN, G. D. (1995). The Weibull distribution, the power law and the instance theory of automaticity. Psychological Review, 102, 751-756.

MCClelland, J. L., \& Rumelhart, D. E. (1981). An interactive activation model of context effects in letter perception: Part I. An account of basic findings. Psychological Review, 88, 375-407.

MCClelland, J. L., \& Rumelhart, D. E. (1985). Distributed memory and the representation of general and specific information. Journal of Experimental Psychology: General, 114, 159-188.

MORTON, J. (1979). Facilitation in word recognition: Experiments causing change in the logogen model. In P. A. Kohlers, M. Wrolstad, \& H. Bouma (Eds.), Processing of visible language (Vol. 1, pp. 259 268). New York: Plenum.

Moses, Y., Ullman, S., \& EdLeman, S. (1996). Generalization to novel images in upright and inverted faces. Perception, 25, 443-461.

Newell, A., \& Rosenbloom, P. S. (1981). Mechanisms of skill acqui- sition and the law of practice. In J. R. Anderson (Ed.), Cognitive skills and their acquisition (pp. 1-55). Hillsdale, NJ: Erlbaum.

Perret, D., Heitanen, J., Oram, M., \& Benson, P. (1992). Organization and function of cells responsive to faces in the temporal cortex. Philosophical Transactions of the Royal Society. London B, 355, 23-30.

Press, W. H., Flannery, B. P., Teukolosky, S. A., \& Vetterling, W. T. (1992). Numerical recipes-the art of scientific computing. Cambridge: Cambridge University Press.

RANER, K. (1994). MacCurveFit [Computer program]. Victoria, Australia: Kevin Raner Software.

RATCLIFF, R. (1979). Group reaction time distributions. Psychological Bulletin, 86, 446-461.

TownSEND, J. T. (1990). Truth and consequences of ordinal differences in statistical distributions: Towards a theory of hierarchical inference. Psychological Bulletin, 108, 551-567.

Valentin, D., Abdi, H., \& Edelman, B. (1997). What represents a face? A computational approach for the integration of physiological and psychological data. Perception, 26, 1271-1288.

VAN ZANDT, T., \& RATCLIFF, R. (1995). Statistical mimicking of reaction time data: Single-process models, parameter variability, and mixtures. Psychonomic Bulletin \& Review, 2, 20-54.

Young, A. W., McWeeny, K. H., HaY, D. C., \& Ellis, A. W. (1986a). Access to identity-specific semantic codes from familiar faces. Quarterly Journal of Experimental Psychology, 38A, 271-295.

Young, A. W., McWeeny, K. H., HaY, D. C., \& Ellis, A. W. (1986b). Matching familiar and unfamiliar faces on identity and expression. Psychological Research, 48, 63-68.

(Manuscript received November 13, 1997; revision accepted for publication March 9, 1999.) 